\title{
Quinolinic Acid Metabolism in the Rat Brain. Immunohistochemical Identification of 3-Hydroxyanthranilic Acid Oxygenase and Quinolinic Acid Phosphoribosyltransferase in the Hippocampal Region
}

\author{
Christer Köhler, ${ }^{1}$ Lars G. Eriksson, ${ }^{1}$ Per R. Flood, ${ }^{2}$ Jon A. Hardie, ${ }^{2}$ Etsuo Okuno, ${ }^{3}$ and Robert Schwarcz ${ }^{3}$ \\ Department of Neuropharmacology, Astra Alab AB, S-151 85 Södertälje, Sweden, ${ }^{2}$ Institute of Anatomy, University \\ of Bergen, N-5000 Bergen, Norway, and ${ }^{3}$ Maryland Psychiatric Research Center, Baltimore, Maryland 21228
}

Quinolinic acid (QUIN) is a potent endogenous excitotoxin, which has been shown to be present in the brain (Wolfensberger et al., 1983). In order to study the cellular localization of QUIN metabolism in the hippocampus, specific antibodies raised against purified rat liver 3-hydroxyanthranilic acid oxygenase (3HAO) and quinolinic acid phosphoribosyltransferase (QPRT), the enzymes directly responsible for QUIN synthesis and catabolism, respectively, were used for immunohistochemical studies in the adult male rat. Cells containing 3HAO immunoreactivity (3HAO-i) were present in all subfields of the hippocampal region, including the area dentata, Ammon's horn, the subicular complex, and the entorhinal area. The highest density of 3HAO-i cells was found in the molecular layer of Ammon's horn and in the hilus of area dentata, while the granular cell layer of area dentata and stratum pyramidale of Ammon's horn contained the lowest number of 3HAO-stained cells. A majority of hippocampal 3HAO-i cells were also stained with monoclonal antibodies against glial fibrillary acidic protein (GFAP) or S-100 protein, suggesting that $3 \mathrm{HAO}-\mathrm{i}$ is present primarily in astrocytes. At the ultrastructural level, 3HAO-i was found to be distributed uniformly throughout the cytoplasm, with intense immunostaining present in the internal and the external layers of the mitochondria.

QPRT-i was detected in 3 morphologically distinct cell types present in all parts of the hippocampus. The total number of QPRT-i cells was lower than that of the 3HAO-i cells. QPRT-I cells were relatively numerous in the molecular and radial layers of Ammon's horn, while they occurred only sporadically in stratum pyramidale of Ammon's horn and in the granular cell layer of area dentata. Many QPRT-i cells stained with antibodies against GFAP and S-100, but the proportion of cells in which QPRT was colocalized with these glial marker proteins was lower than that for 3-HAO-i cells. At the ultrastructural level, 2 types of QPRT-i glial cells were detected. The smaller cell type had a diffuse cytoplasmic staining, while the larger cell type, which also contained glial filaments, showed diffuse cytoplasmic staining and intense staining of lysosomal structures. The observation that $3 \mathrm{HAO}$ and QPRT only partially coexist in hippocampal glial cells

\footnotetext{
Received June 16, 1987; revised Sept. 22, 1987; accepted Sept. 23, 1987.

This work was supported by USPHS Grants NS 16102 and NS 20509.

Correspondence should be addressed to Christer Köhler at the above address.

Copyright (C) 1988 Society for Neuroscience $0270-6474 / 88 / 030975-13 \$ 02.00 / 0$
}

suggests that while synthesis and catabolism of QUIN may occur in the same glial cells, catabolism of QUIN can also take place in cells lacking the synthetic enzyme. These findir.gs may have implications for the hypothesis that QUIN is a potential pathogen in neurodegenerative diseases afflicting the hippocampal region.

The central role of the hippocampal formation in the initiation and/or propagation of seizure phenomena has been recognized for several decades (see Falconer et al., 1955, and DelgadoEscueta et al., 1986 for review) and descriptions of selective hippocampal neuronal loss (Ammon's horn sclerosis) in temporal lobe epileptics reach back even to the 19th century (Sommer, 1880). Until relatively recently, however, only sporadic attention has been paid to the neurochemical basis and correlates of seizures. From these studies, 2 prominent hypotheses have emerged; they propose either disinhibition (namely, a lack of $\gamma$-aminobutyric acid, or GABA) or overexcitation by glutamate or its congeners as constituting the single most important principle of epileptogenesis (Delgado-Escueta et al., 1986; Schwarcz and Ben-Ari, 1986). Supportive evidence for both views has been gathered primarily in the hippocampus and other limbic brain areas and in regions of the cerebral cortex.

The current interest in a possible pathogenic role of endogenous excitatory amino acids in epilepsy stems from the realization that hyperphysiological synaptic concentrations of these compounds not only precipitate several physiological events that are characteristic for ictal episodes, but also cause, via excitotoxic mechanisms, neuronal loss in the hippocampus that is highly reminiscent of that observed in Ammon's horn sclerosis. Of the 3 established subtypes of excitatory amino acid receptors (Foster and Fagg, 1984), the $N$-methyl-D-aspartate (NMDA) site is most likely preferentially involved in the mediation of seizure phenomena. Thus, specific antagonists of the NMDA receptor not only possess powerful anticonvulsant properties in a spectrum of experimental models of epilepsy, but also prevent the occurrence of excitotoxic cell death induced by NMDA agonists (Croucher et al., 1982; Schwarcz et al., 1982, 1984; Wong et al., 1986).

Several endogenous neuroexcitatory compounds have been shown to interact with the NMDA receptor and are therefore viable candidates as pathogens in seizure disorders. Glutamate and aspartate, present in the brain in millimolar concentrations, not only lack specificity for the NMDA site, but are also very weak convulsants and neurotoxins, probably owing to their rap- 
id removal from the synapse by transport processes and metabolism (Köhler and Schwarcz, 1981; Foster and Fagg, 1984). In contrast, quinolinic acid (QUIN), a quantitatively minor, yet regular, constituent of rodent and human brain (Wolfensberger et al., 1983; Moroni et al., 1984), is a potent convulsant (Lapin, 1981; Lapin et al., 1982; Schwarcz et al., 1984) likely to act exclusively via the NMDA receptor (Stone and Perkins, 1981). Notably, there exists pronounced regional variability in both the excitatory and toxic effects of QUIN (Perkins and Stone, 1983; Schwarcz and Köhler, 1983), with the hippocampal formation being particularly sensitive.

The investigation of QUIN neurobiology has recently been greatly facilitated by the availability of specific antibodies against the 2 enzymes directly responsible for QUIN metabolism, 3-hydroxyanthranilic acid oxygenase (3HAO) and quinolinic acid phosphoribosyltransferase (QPRT; Foster et al., 1985, 1986; Okuno and Schwarcz, 1985; Okuno et al., 1987). In view of the possible role of QUIN in seizure disorders (Schwarcz et al., 1986) and other neuropsychiatric disorders involving the hippocampus (Schwarcz and Meldrum, 1985), we have now therefore studied the morphological characteristics of the normal rat hippocampal QUIN system by immunocytochemistry using anti$3 \mathrm{HAO}$ and -QPRT antibodies.

\section{Materials and Methods}

Preparation of tissue. Male Sprague-Dawley rats (Alab AB, Sollentuna, Sweden; $200 \mathrm{gm}$ ) were deeply anesthetized (Mebumal; ACO, $60 \mathrm{mg}$ $\mathrm{kg}^{-1}$, I.P.) and perfused through the ascending aorta with $50 \mathrm{ml}$ saline $\left(22^{\circ} \mathrm{C}\right)$ followed by $400 \mathrm{ml}$ of a fixative containing paraformaldehyde: lysine : periodate prepared as described by MacLean and Nakane (1974). The perfused brains were postfixed for no more than $4 \mathrm{hr}$ and then transferred to PBS containing $10 \%(\mathrm{wt} / \mathrm{vol})$ sucrose. After $2 \mathrm{~d}$, the brains were cut on a freezing microtome and horizontal sections ( $30 \mu \mathrm{m}$ thick) were collected in wells containing PBS.

Antibodies. The anti-3HAO and anti-QPRT antibodies were raised in rabbits against purified rat liver $3 \mathrm{HAO}$ and QPRT (Okuno and Schwarcz, 1985; Okuno et al., 1987). The antiserum against glial fibrillary acidic protein (GFAP; Dako Patts, Copenhagen, Denmark) was produced in rabbits against GFAP purified from human astrocytoma. In addition, a monoclonal anti-GFAP antibody (BioGenex) was used in double-staining experiments. Monoclonal antibody against the S-100 protein was kindly donated by Dr. B. Boss, Salk Institute, San Diego, CA

Immunohistochemical procedure. The brain sections were thoroughly rinsed in PBS and, floating free, were incubated in anti-3HAO (diluted $1: 8000$ in PBS containing $0.2 \%$ Triton $X-100$ and $1 \%$ normal goat serum), anti-QPRT (diluted 1:6000), anti-GFAP (both polyclonal and monoclonal antiserum, diluted 1:12,000), or anti-S-100 (diluted 1:6000) antibodies for $7 \mathrm{~d}$.

Each antigen-antibody complex was made visible through the avidinbiotin-complex (ABC) method of Hsu et al. (1981), using a commercially available $\mathrm{ABC}$ kit (Vector Laboratories, Burlingame, $\mathrm{CA}$ ) with 3',3'-diaminobenzidine (DAB; Sigma Chemical Co., St. Louis, MO) as a chromogen. While most sections were defatted and coverslipped in Permount (Histolab, Gothenburg, Sweden), others were processed for thionin staining. In some experiments, the sections were treated with $0.2 \%$ osmium tetroxide to enhance the intensity of the $\mathrm{DAB}$ reaction product (Johansson and Backman, 1983).

Double-staining experiments. In the double-staining experiments, which were aimed at localizing 2 antigens in the same tissue section, the incubations were performed in a cocktail containing 2 antibodies (anti-rabbit 3HAO or QPRT antiserum together with either monoclonal GFAP or monoclonal S-100 antiserum). Localization of 3HAO and QPRT-i in GFAP cells was made by first visualizing either $3 \mathrm{HAO}$ or QPRT with DAB, which yielded a brown reaction product. The sections were then washed extensively, exposed to $4 \%$ paraformaldehyde for 1 $\mathrm{hr}$, and thereafter reacted with a rabbit anti-mouse $A B C$ kit with 4-chloronaphtol (Sigma) as the chromogen, which yielded a blue-black color. The rabbit anti-mouse IgG was preadsorbed with purified rabbit IgG. In the experiments in which colocalization with S-100 was ex- amined, the primary antibody was made visible by either goat antirabbit fluorescein (for 3HAO or QPRT) or rabbit anti-mouse rhodamine (for S-100) isothyiocyanate-conjugated IgG (Miles-Yeda, Rehovot, Isracl).

Control experiments. Control experiments with $3 \mathrm{HAO}$ and QPRT antisera included incubation of sections in preimmune serum from the same rabbits that generated the antisera or incubation in antiserum preadsorbed in liquid phase with the respective pure antigens. Neither of these procedures resulted in staining of cells in the tissue sections.

Electron microscopy. Rats were perfusion-fixed according to the $\mathrm{pH}$ shift technique of Berod et al. (1981), using $0.05 \%$ glutaraldehyde in addition to the $4 \%$ formaldehyde in the second fixative. After $10 \mathrm{~min}$ perfusion through the ascending aorta, the brain was isolated and postfixed for another $4 \mathrm{hr}$ in the same fixative before transfer to PBS. The next morning, $100 \mu \mathrm{m}$ cross sections of the hippocampal formation were cut on a Lancer Vibratome. These were processed for indirect peroxidase immunostaining, as described above, with the following modifications: the primary antibodies were diluted between 1:1000 and 1:2000 and used for $1 \mathrm{~d}$ only. No detergents were added. After the DAB reaction, the sections were treated with $0.01 \% \mathrm{OsO}_{4}$, rinsed in PBS, incubated in $0.5 \%$ thiosemicarbazide, rinsed, postfixed for $1 \mathrm{hr}$ in $1 \%$ $\mathrm{OsO}_{4}$, rinsed in distilled water, stained by $1 \%$ uranyl acetate in water, dehydrated in increasing concentrations of ethanol, and embedded in Epon. Sections, $2 \mu \mathrm{m}$ thick, were cut on a LKB ultramicrotome, examined, and photographed through a Zeiss photomicroscope, reembedded in Epon, and resectioned as ultrathin sections. These were mounted on carbon and formar-coated copper grids and examined in a Philips EM300 transmission electron microscpe without further staining.

\section{Results}

\section{Light-microscopic observations}

$3 H A O$-immunoreactive cells. A large number of cells in the hippocampal region were stained with $3 \mathrm{HAO}$ antiserum (Figs. 1, $A-D ; 2, A, C)$. 3HAO-immunoreactive (3HAO-i) cells ranged in size between 5 and $10 \mu \mathrm{m}$ across their cell bodies. All cells had wcll-stained processes, which in some cases ramificd in all directions, while in other cases showed a unipolar orientation (Fig. $3 B$ ). The light-microscopic appearance of $3 \mathrm{HAO}-\mathrm{i}$ cells suggests that they are glial rather than neuronal in nature, a conclusion supported by double-labeling experiments with glial markers (see below).

3HAO-i cells were present in all subfields of the hippocampal region, and individual $3 \mathrm{HAO}-\mathrm{i}$ cells were encountered in each lamina of each hippocampal subfield (Figs. 1, $A-D ; 2, A, C$ ). However, a clear difference in the total number of $3 \mathrm{HAO}-\mathrm{i}$ cells existed between individual laminae. Thus, cell counts performed in horizontally cut sections of the dorsal one-third of the hippocampus showed that, within the area dentata and Ammon's horn, the granular cell layer and stratum pyramidale, respectively, contained relatively few $3 \mathrm{HAO}$-stained cells, while the molecular layers of both subfields were rich in 3HAO-i glial cells (Table 1). Similarly, the molecular layer of the subiculum harbored more $3 \mathrm{HAO}-\mathrm{i}$ cells than did the pyramidal layer of this structure (Table 1, Fig. 1C). In the entorhinal area, all layers, with the exception of layer 1 , were rich in $3 \mathrm{HAO}-\mathrm{i}$ glial cells (Fig. 1A). There existed no apparent gradient in the number of $3 \mathrm{HAO}-\mathrm{i}$ cells along the longitudinal axis of the hippocampus. When all 3HAO-i cells in each region were counted, the area dentata was found to contain more cells than any of the other subfields (Table 1). Each hippocampal subfield was far richer in 3HAO-i than in QPRT-i glial cells with regard to the total number of cells (Fig. 1, $A-H$; Table 1).

QPRT-immunoreactive cells. Specific QPRT-i was detected within a large number of small cells scattered throughout all layers of every hippocampal subfield (area dentata, Ammon's horn, subicular complex, and entorhinal area; Figs. 1, E-H;2, $B, D)$. Some QPRT-i cells were situated among the myelinated 


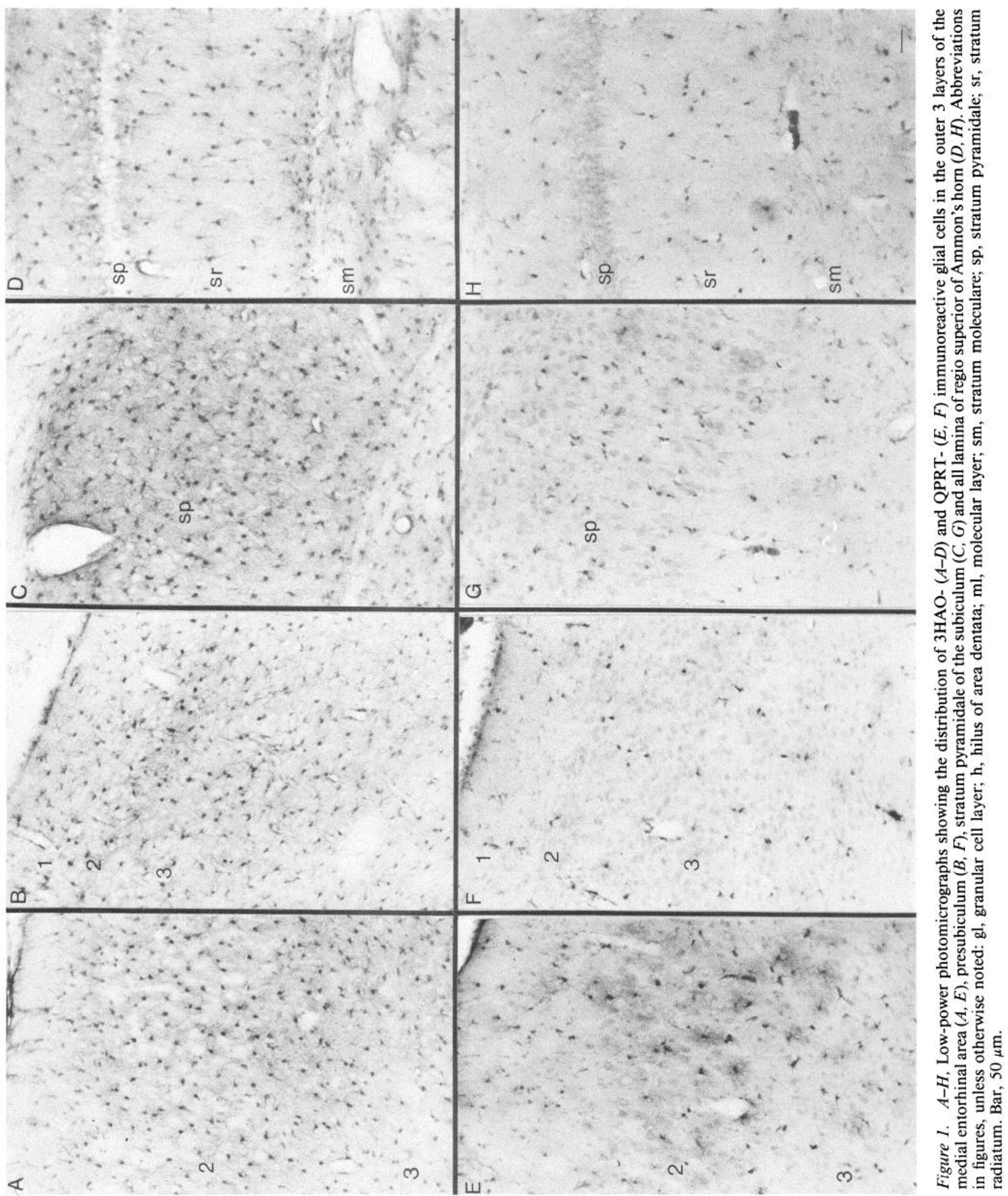



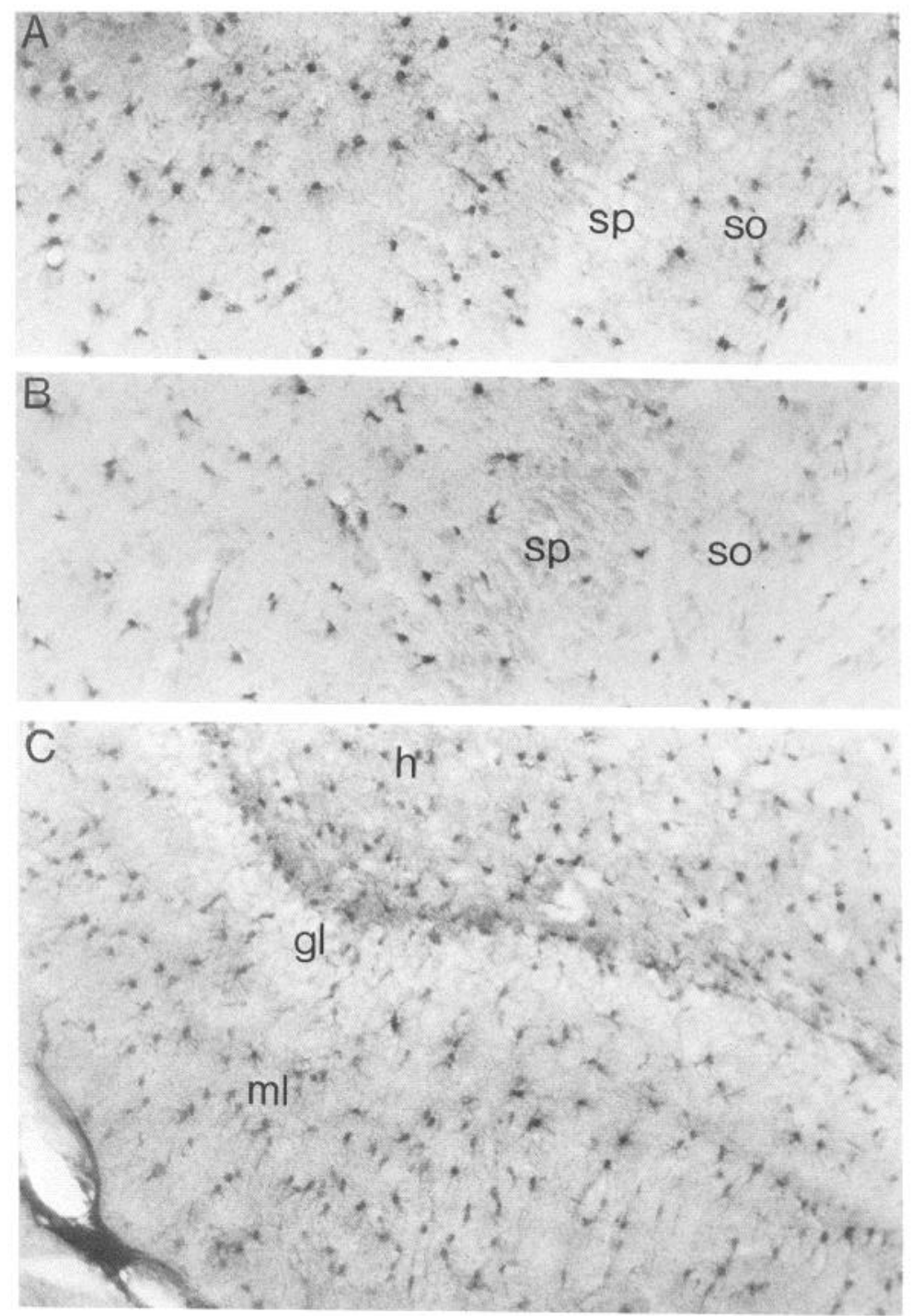

Figure 2. $A-D$, Low-power photomicrographs of $3 \mathrm{HAO}-(A, C)$ and QPRT- $(B, D)$ immunoreactive glial cells in the regio inferior of Ammon's horn and area dentata $(C, D)$. Bar, 50 $\mu \mathrm{m}$.

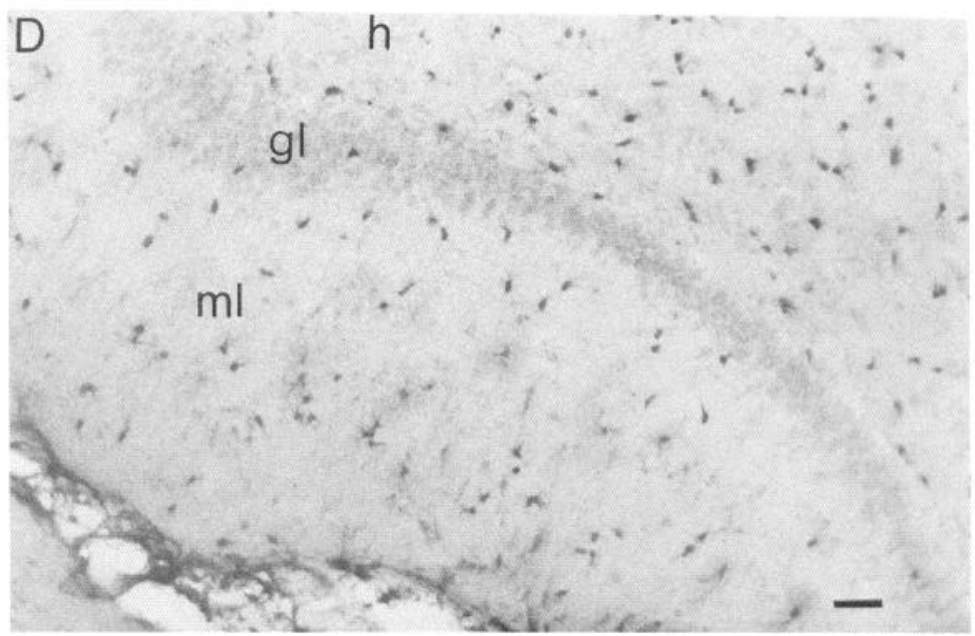

fibers of the alveus, angular bundle, and fornix-fimbria. They were small in size (cell body diameter, 5-12 $\mu \mathrm{m}$ ) and possessed round or oval cell bodies. Most of the QPRT-i cells had short, highly tortuous processes ramifying in all directions (Fig. $3 \mathrm{~A}$ ), while some cells had few and relatively poorly developed ones
(Fig. $3 G$ ). Both light- and electron-microscopic (see below) observations suggest that these QPRT structures are glial cells. With one exception (the area dentata), there existed no clear difference in the density of QPRT-i cells between the individual hippocampal subfields (Table 1). When all cells were counted, 

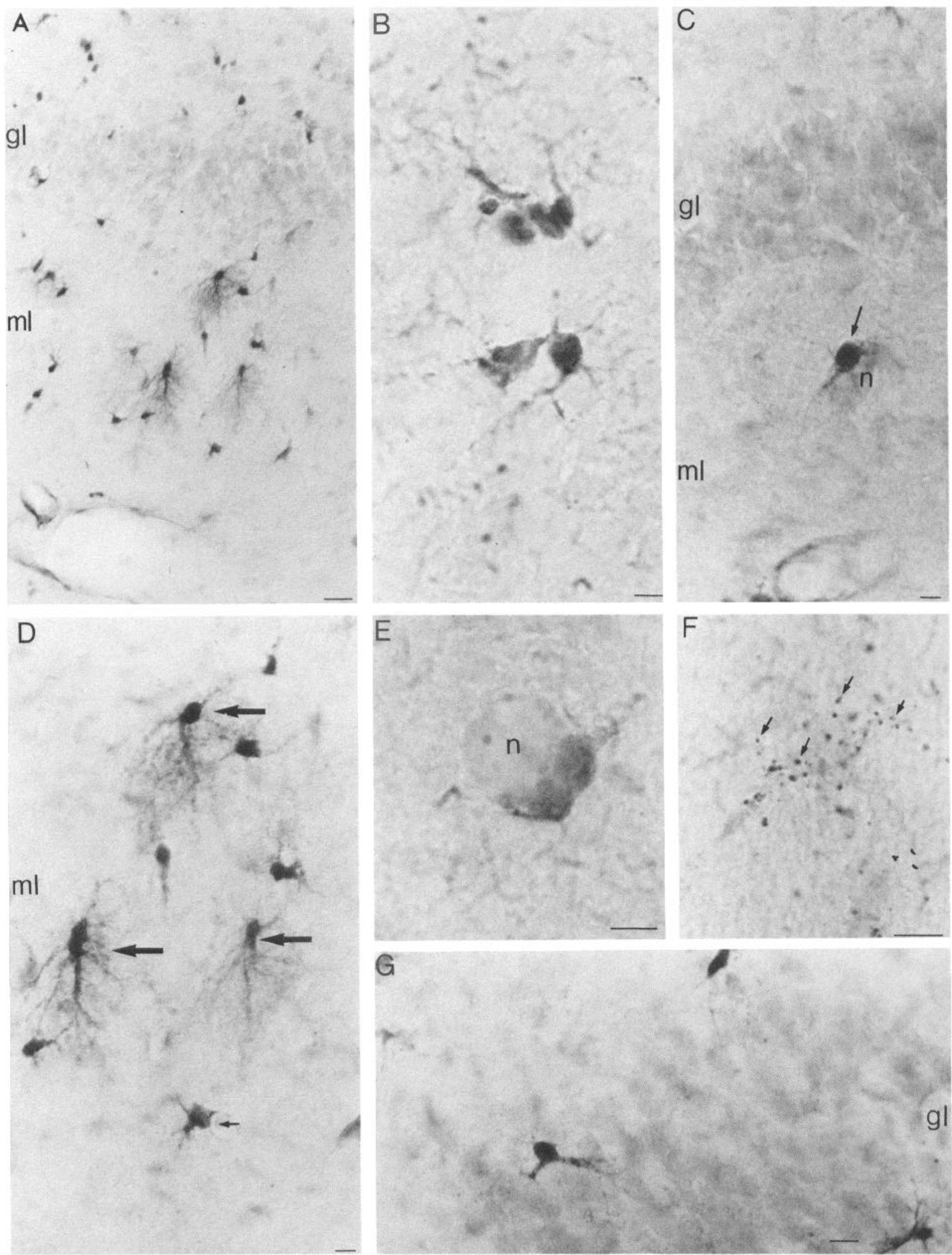

Figure 3. $A-G$, Photomicrographs showing QPRT-i cells of different morphological types in the molecular layer of area dentata $(A, C, D)$, hilus $(E)$ and granular cell layer $(G)$. Photomicrograph in $F$ shows QPRT-i punctate structures (small arrows) on a presumed astroglial cell in the molecular layer of area dentata. In $C$ and $E$ QPRT-i glial cells are shown in close association with neuronal somata $(n)$, suggesting direct contact between QPRT-i cells and neurons. Arrows in D mark large QPRT-i glial cells in the molecular layer. Bar, $10 \mu \mathrm{m}$. 


\begin{tabular}{|c|c|c|}
\hline & $3 \mathrm{HAO}$ & QPRT \\
\hline \multicolumn{3}{|l|}{ Area dentata } \\
\hline Molecular layer & $395 \pm 55(14.6 \pm 0.8)$ & $182 \pm 11(5.2 \pm 0.3)$ \\
\hline Granular cell layer & $95 \pm 5 \quad(9.4 \pm 0.5)$ & $20 \pm 4 \quad(1.2 \pm 0.2)$ \\
\hline Hilus & $350 \pm 33(13.8 \pm 0.9)$ & $103 \pm 10(9.0 \pm 0.5)$ \\
\hline \multicolumn{3}{|l|}{ Regio inferior } \\
\hline Stratum oriens & $215 \pm 34(13.8 \pm 0.9)$ & $46 \pm 3 \quad(5.8 \pm 0.4)$ \\
\hline Stratum pyramidale & $67 \pm 9 \quad(4.5 \pm 0.4)$ & $40 \pm 3 \quad(1.2 \pm 0.2)$ \\
\hline Stratum lucidum & $158 \pm 17(10.7 \pm 0.5)$ & $35 \pm 15(6.2 \pm 0.6)$ \\
\hline Stratum radiatum & $163 \pm 22(14.7 \pm 0.6)$ & $39 \pm 16(3.6 \pm 0.6)$ \\
\hline \multicolumn{3}{|l|}{ Regio superior } \\
\hline Stratum oriens & $195 \pm 39(11.5 \pm 0.6)$ & $42 \pm 4 \quad(4.7 \pm 0.4)$ \\
\hline Stratum pyramidale & $47 \pm 4 \quad(5.0 \pm 0.6)$ & $19 \pm 5 \quad(0.7 \pm 0.2)$ \\
\hline Stratum radiatum & $235 \pm 12(8.6 \pm 0.4)$ & $99 \pm 11(4.1 \pm 0.3)$ \\
\hline Stratum moleculare & $295 \pm 30(19.3 \pm 1.1)$ & $101 \pm 15(4.2 \pm 0.3)$ \\
\hline \multicolumn{3}{|l|}{ Subiculum } \\
\hline Stratum pyramidale & $185 \pm 29(12.6 \pm 0.6)$ & $79 \pm 14(4.2 \pm 0.4)$ \\
\hline Stratum moleculare & $231 \pm 32(16.7 \pm 0.6)$ & $63 \pm 3(4.8 \pm 0.4)$ \\
\hline \multicolumn{3}{|l|}{ Presubiculum } \\
\hline Layer 1 & $89 \pm 30(7.3 \pm 1.3)$ & $19 \pm 10(3.4 \pm 0.3)$ \\
\hline Layer 2 & $540 \pm 65(15.3 \pm 1.2)$ & $36 \pm 5 \quad(3.1 \pm 0.3)$ \\
\hline Layers 3-6 & n.d. $\quad$ n.d. & n.d. n.d. \\
\hline \multicolumn{3}{|l|}{ Entorhinal area } \\
\hline Layer 1 & $65 \pm 18(5.4 \pm 0.4)$ & $56 \pm 6 \quad(4.4 \pm 0.3)$ \\
\hline Layer 2 & $246 \pm 38(15.3 \pm 1.0)$ & $52 \pm 9 \quad(6.0 \pm 0.3)$ \\
\hline Layer 3 & $241 \pm 15(16.4 \pm 0.6)$ & $46 \pm 10(5.9 \pm 0.3)$ \\
\hline Layers 4-6 & $168 \pm 14(16.0 \pm 1.0)$ & $35 \pm 3 \quad(5.4 \perp 0.3)$ \\
\hline
\end{tabular}

The values represent cell counts performed in 4 hippocampi of 2 rats. n.d., Not determined.

the largest number of QPRT-i cells was found in the area dentata and regio superior. The number and the density of QPRT-i cells differed between individual laminae of Ammon's horn, area dentata, subicular complex, and entorhinal area. As shown in Figures 1 and 2 and Table 1, the pyramidal and granular cell layers of Ammon's horn and the area dentata (both of which contain densely packed neuronal somata) were relatively poor in QPRT-i glial cells. Similarly, stratum lucidum of regio inferior contained few QPRT-i cells, while the molecular layer and the hilus of area dentata were found to be very rich in stained glial cells (Table 1).

On the basis of morphological criteria, 3 principal cell types containing QPRT-i could be identified at the light-microscopic level. Each of these had unique distribution patterns within the hippocampus. Thus, the majority of QPRT-i cells were small $(5-7 \mu \mathrm{m})$, with one or 2 major process(es) extending short distances from the cell body (Fig. 3G). In many instances, such glial cells had processes oriented towards a neuronal perikaryon, and occasionally QPRT-i "endfeet" were present on the soma surface of the neuron (Fig. 3, $C, G$ ). These glial cells were found in all parts and layers of the hippocampal region. Another glial cell type had an oval cell body with few, short processes. Frequently, these glial cells were situated in close association with neuronal somata, suggesting direct contacts between the QPRT-i glial cell body and the neuronal perikaryon (Fig. 3, $C, E$ ). Glial cells of this type were present in large numbers in the hilus of area dentata. Glial cells of the third group were slightly larger (approximately $12 \mu \mathrm{m}$ ). Their cell bodies were oval with relatively straight and widely ramifying processes extending from one end of the cell soma (Fig. 3, $A, D$ ). These glial cells were found almost exclusively in the molecular layer of area dentata. Their main processes always extended towards the pial surface of this layer (Fig. 3D).

Many of the QPRT-i glial cells had endfeet seemingly in contact with neuronal perikarya. At the light-microscopic level, numerous QPRT-i glial cells had processes extending over neuronal somata, and punctate QPRT-i structures were visible on the surface of granule and pyramidal cells, as well as among cells in the hilus of area dentata. In the molecular layer of area dentata, QPRT-i punctate structures appeared to be present on some QPRT-i (not shown), as well as on QPRT-negative glial cells (Fig. $3 F$ ).

\section{Electron microscopy}

$3 H A O-i$ cells. In semithin sections of material processed for electron microscopy, the $3 \mathrm{HAO}-\mathrm{i}$ cells were similar to those described above from the material processed for light microscopy (Fig. 4). Although the number, size, and pattern of cytoplasmic processes varied slightly between different hippocampal areas and laminae, all immunoreactive cells seemed to belong to the same cell type. Their ultrastructure, as described below, was also the same for all areas included in this study. The $3 \mathrm{HAO}-\mathrm{i}$ cells mostly revealed a weakly stained nucleus of 5-10 $\mu \mathrm{m}$ diameter, surrounded by an intensely stained cytoplasmic 
layer of $0.5-4 \mu \mathrm{m}$ width, from which one or several equally positively stained cytoplasmic processes of $0.5-3 \mu \mathrm{m}$ diameter radiated (Fig. 4). Some of these immunoreactive processes could be traced to their termination as perivascular or perineuronal endfeet. In the electron microscope, the immunoprecipitate seemed fairly uniformly distributed throughout the cytosol of the 3HAO-i cells (Fig. 4). The interior of most membrane-enclosed cytoplasmic structurcs, such as lysosomc-like bodies with heterogeneous content, Golgi apparatus and vesicles, multivesicular bodies, and cisterns of the endoplasmic reticulum, was unstained. The mitochondria revealed an intensely stained internal as well as external boundary membrane, while the cristae were weakly stained only in patches. The matrix compartment was always unstained. Filament bundles were usually present in the 3HAO-i cell bodies and their processes; however, the finer details of these filaments were obscured by the immunoprecipitate (Fig. 4).

QPRT-i cells. In semithin sections (Fig. 5), 2 kinds of immunoreactive cells could be discerned. Some small cells with few processes mostly revealed a diffuse cytoplasmic and sometimes nuclear immunostaining. Some larger cells with more cytoplasmic processes revealed intensely stained cytoplasmic granules in addition to a weaker diffuse staining. Immunoreactive granulcs werc scattered throughout the neuropil in processes unidentifiable at the light-microscopic level. In the electron microscope, the diffuse cytoplasmic immunostaining of the smaller cell type (Fig. 5) was confined to patches in the cytosol compartment, sparing the interior of mitochondria, lysosomes, Golgi and endoplasmic reticulum cisterns. These cells were found to contain neither filament bundles nor a prominent endoplasmic reticulum. The larger cell type revealed a weak cytosol immunostaining, sparing most of the membrane-enclosed organelles except for some globular lysosome-like bodies with homogeneous content, which were intensely immunoreactive (Fig. 5). These cells usually had a large, lightly stained nucleus, prominent filament bundles in their cytoplasmic processes, and some areas of watery cytoplasm (swelling artefact), both representative of fibrous astrocytes.

The granular immunoreactivity of the neuropil turned out to represent cytoplasmic islands, probably of glial origin, related to the cytoplasmic processes of the above-described cell types. Pre- and postsynaptic neuronal components were mostly immunonegative.

\section{Double-staining experimenis}

Double-staining of tissue sections with polyclonal antibodies to either QPRT or 3HAO and monoclonal antibodies to GFAP or $\mathrm{S}-100$ confirmed that both enzymes were associated with astrocytic glial cells (Fig. $6, A-D$ ). Thus, numerous cells in the hippocampus contained both QPRT and GFAP or S-100-i (Fig.
$6 A)$. Such double-labeled cells occurred in all hippocampal subfields, as well as among myelinated fiber tracts (e.g., fornixfimbria). Of importance, however, was that not all GFAP- or S-100-positive cells contained QPRT-i (Fig. 6, B, D), and numerous QPRT-i cells were found that lacked either GFAP or S-100 immunostaining (Fig. 6D). While GFAP-i cells lacking QPRT-i were noted in all parts of the hippocampus, QPRT-i cells lacking GFAP or S-100 were particularly frequently encountered in the hilus of the area dentata. Interestingly, QPRT-i glial cells of this type often belonged to the type of cell with few processes described above (Fig. $3 E$ ). Analysis of sections doublestained with 3 HAO and GFAP or S-100 showed that virtually every 3HAO-i cell also contained GFAP and/or S-100 (Fig. 7). Still, individual GFAP- and S-100-positive glial cells lacking $3 \mathrm{HAO}-\mathrm{i}$ could occasionally be found in all hippocampal subfields.

\section{Discussion}

The present study has shown that 3HAO- and QPRT-immunoreactivities are present in glial cells in the hippocampal region of the male rat. These findings were based on the use of specific antibodies raised against purified rat liver $3 \mathrm{HAO}$ and QPRT, respectively (Okuno and Schwarcz, 1985; Okuno et al., 1987). The fact that no staining was obtained in tissue sections incubated with anti-3HAO or anti-QPRT antibodies preadsorbed with the respective antigen indicates that the staining detected in hippocampal cells represents the authentic enzymes. $3 \mathrm{HAO}$ and QPRT are responsible for the synthesis and catabolism, respectively, of QUIN in peripheral tissues (Nishizuka and Hayaishi, 1963; Gholson et al., 1964) and in the brain (Foster et al., 1985, 1986). By using immunocytochemical methods, we have now been able to analyze in more detail the identity of cells containing $3 \mathrm{HAO}$ and QPRT and their distribution within the hippocampal region.

Inspection of both light- and electron-microscopic material suggests that the population of glial cells staining for $3 \mathrm{HAO}$ is morphologically more homogeneous than that stained for QPRT. Double-staining experiments with anti-3HAO and anti-GFAP or anti-S100 antibodies on the same tissue sections demonstrated that most 3HAO-i cells also contain GFAP and/or S-100-i. Electron-microscopic analysis also provided evidence for the presence of glial filaments in most $3 \mathrm{HAO}-\mathrm{i}$ cells. By contrast, double-staining experiments using QPRT antibodies showed that the proportion of QPRT-i cells containing GFAP-i, S-100$\mathrm{i}$, or glial filaments was lower than that observed for $3 \mathrm{HAO}-\mathrm{i}$ cells. These observations indicated that most $3 \mathrm{HAO}-\mathrm{i}$, but not a majority of QPRT-i, is contained in astrocytes.

The exact identity of the QPRT-i cells lacking GFAP-i is unclear at present, hut protoplasmic astrocytes, as well as oligodendrocytes, are likely candidates (Peters et al., 1976). In fact,

\footnotetext{
Figure 4. $A-F$, Corresponding light $(A, D)$ and electron micrographs $(B, E)$ of two 3-HAO-positive cells from the entorhinal cortex $(A-E)$ and hilus of fascia dentata $(F)$. The immunoprecipitate decorates all cytoplasmic structures except the interior of most membrane-bound organelles, like endoplasmic reticulum, Golgi apparatus, lysosome-like bodies, and the matrix compartment of mitochondria $(C, F)$. The intracristal space of mitochondria, however, is immunoreactive $(C)$. $g f$, glial filaments, $m$, mitochondria, $t l$, telolysosome-like bodies, $m v$, multivesicular body, $n$, nucleus.
}

Figure 5. $A-F$, Corresponding light $(A-D)$ and electron micrographs $(B, E)$ of QPRTase-positive cells from the molecular layer $(A-C)$ and hilus $(D-E)$ of fascia dentata. Note the size difference between the 2 immunoreactive cells $(A-D)$. $C$, Detail of an immunoreactive lysosome-like body found in an QPRTase-positive cell similar to the one shown in $B . F$, Detail of the diffuse cytoplasmic immunostaining of the cell shown in $E$. $g$, Golgi apparatus. Otherwise, same labeling as in Figure 4. 


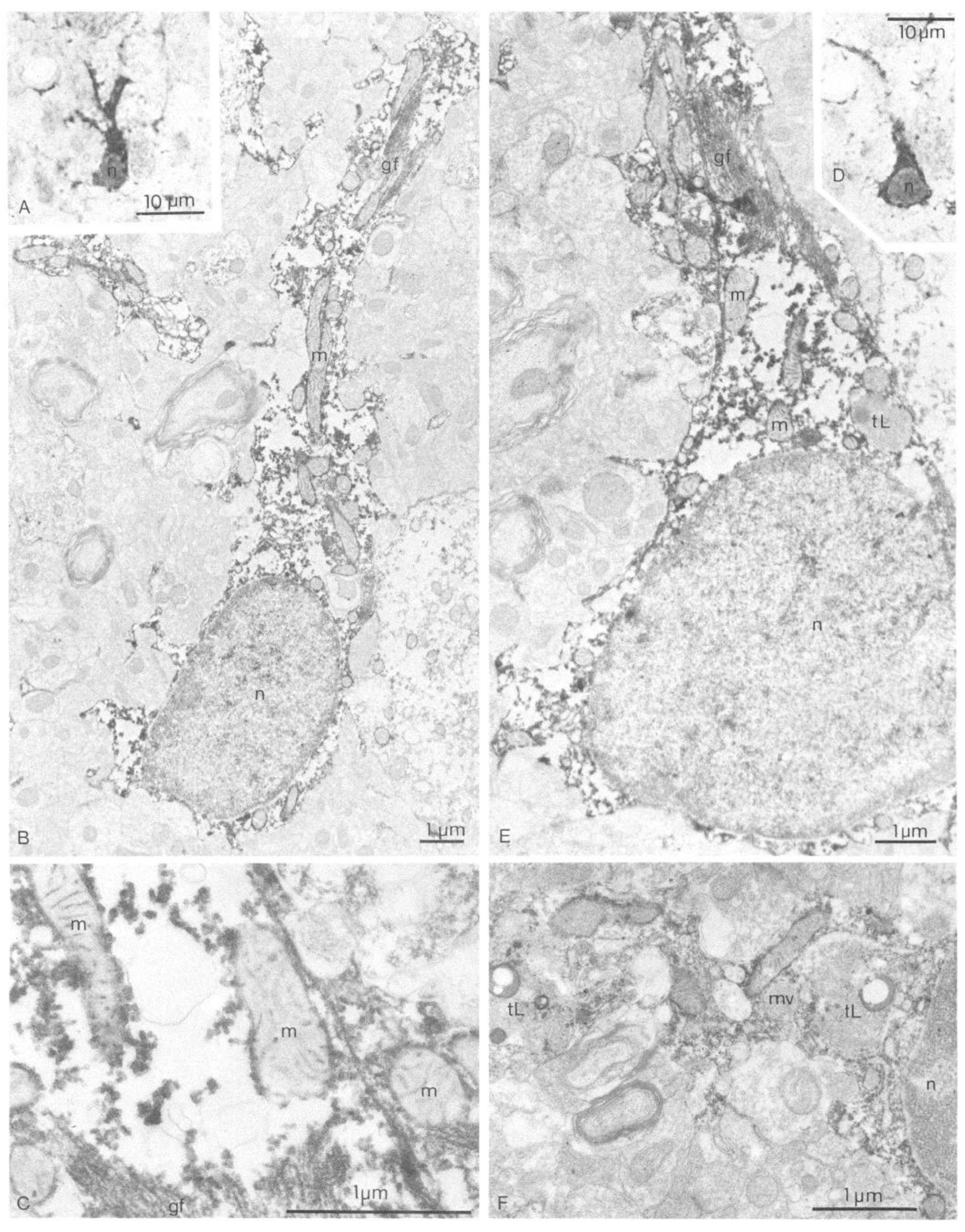




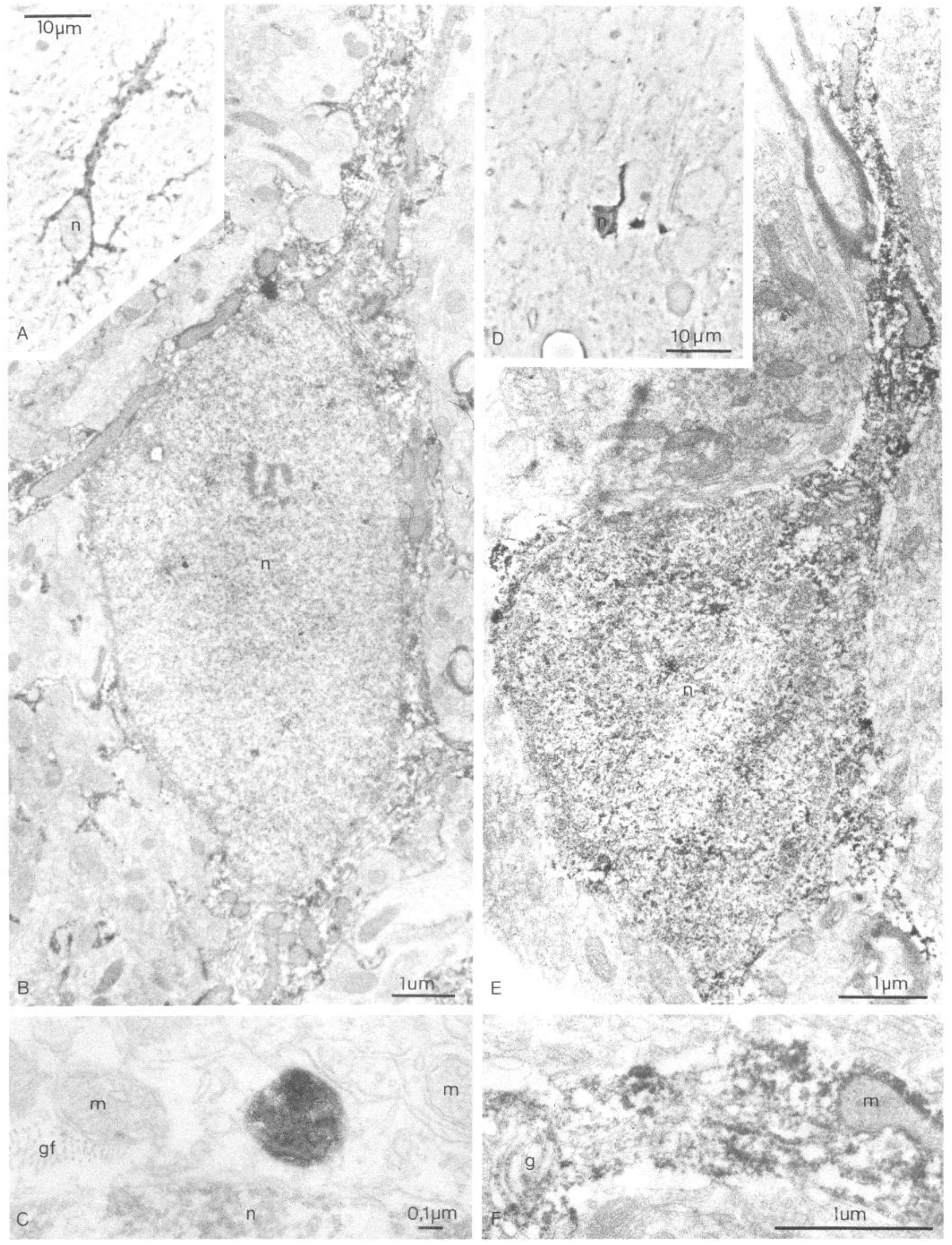




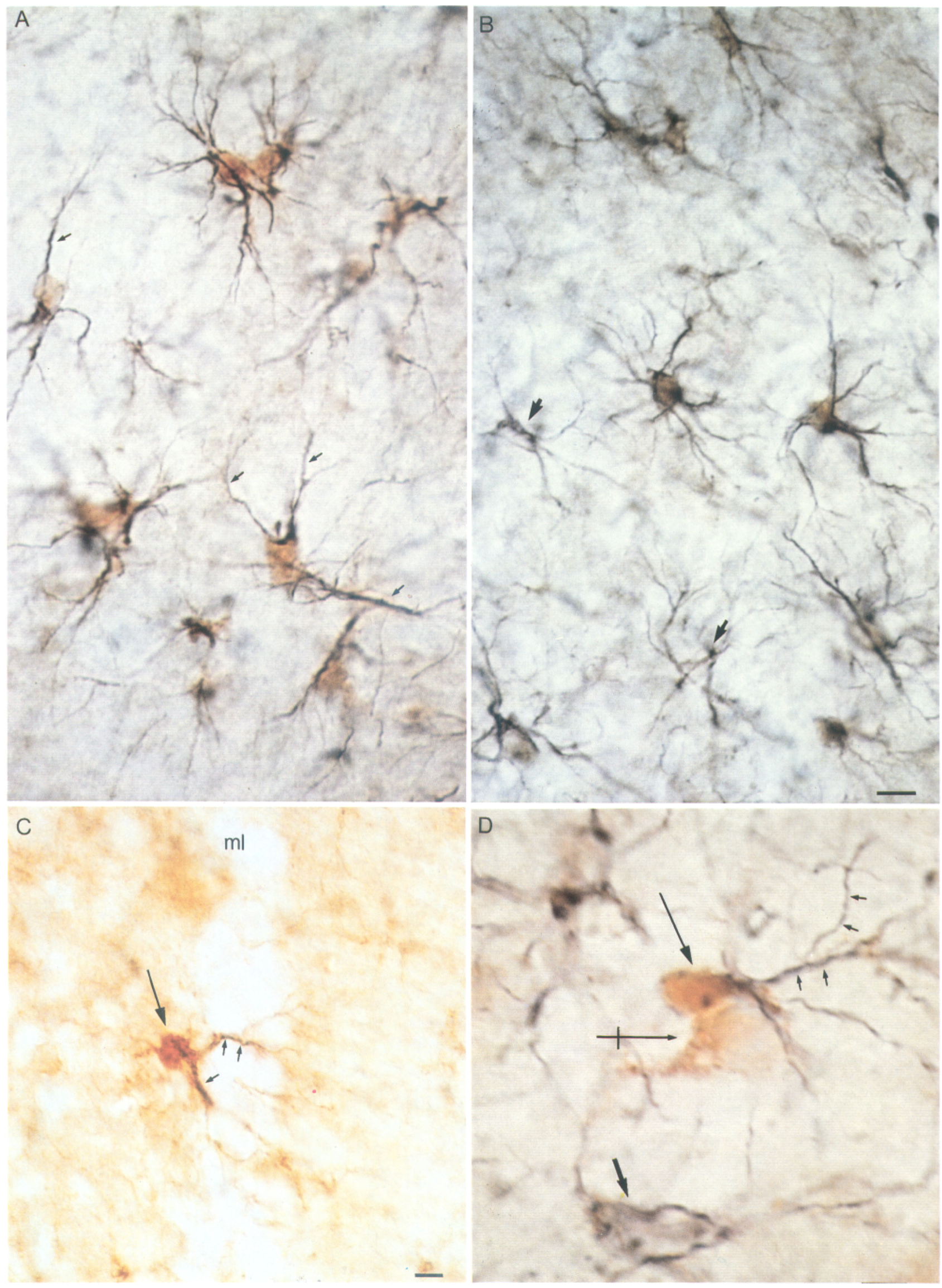



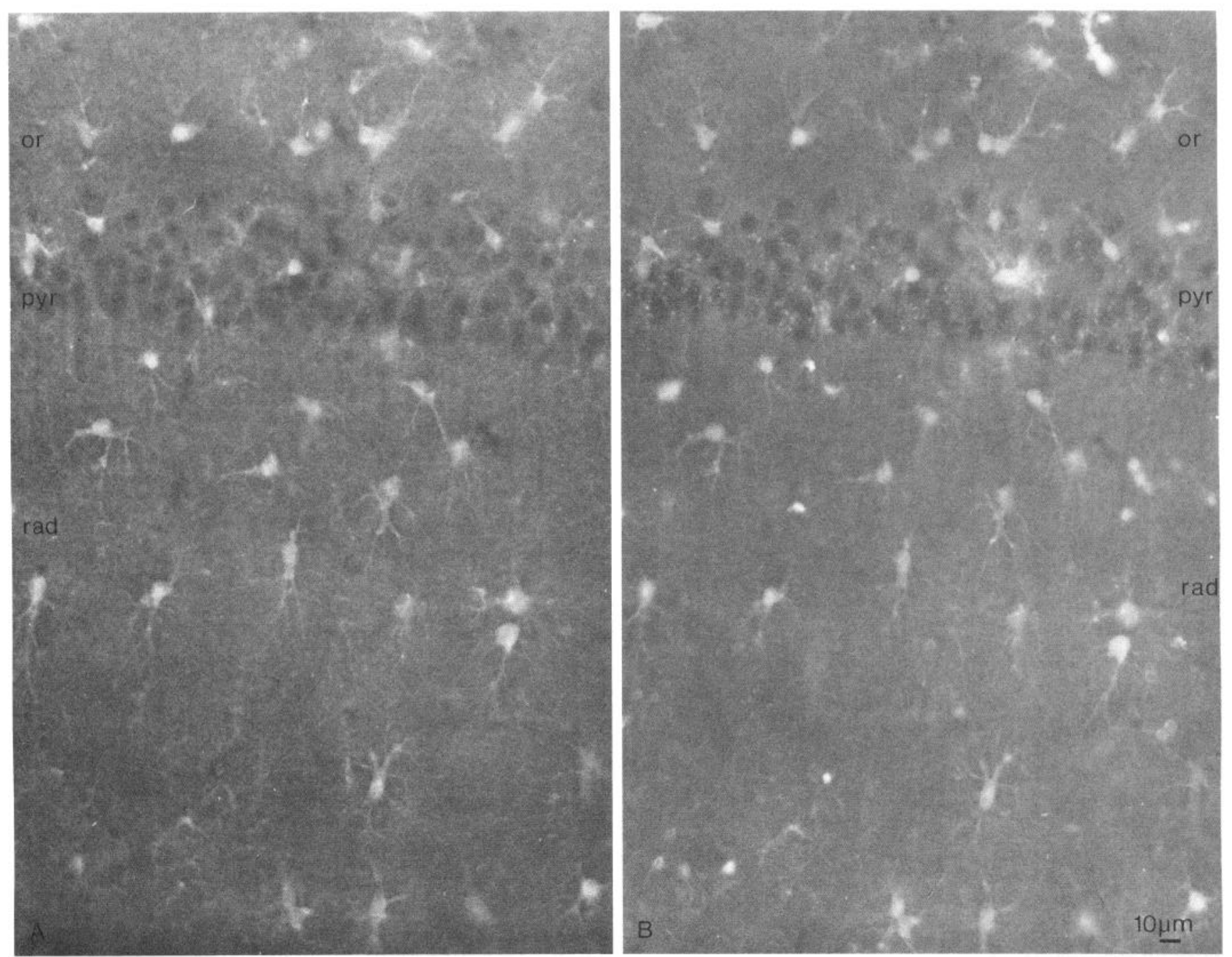

Figure 7. $A, B$, Double-labeled immunofluorescence pictures of hippocampal subfield CA1 from a $25 \mu \mathrm{m}$ frozen section incubated in $(A)$ rabbit anti-3-HAO antibody diluted 1000 times, visualized by FITC-conjugated goat-anti rabbit antibody, and $(B)$ mouse monoclonal anti-S-100 antibody in 2000 times dilution, visualized by rhodamin-conjugated goat anti-mouse antibody. or, stratum oriens, pyr, stratum pyramidale, rad, stratum radiatum. All 3HAO-positive cells are also positive to $\mathrm{S}-100$. Bar, $10 \mu \mathrm{m}$.

the pericellular QPRT-i glial cells detected in the hilus that did not stain for GFAP may represent a type of pericellular or satellite oligodendrocyte described in light- and electron-microscopic studies (see Peters et al., 1976; Vaughan, 1984). In a previous study (Köhler et al., 1987), we showed that QPRT-i is associated with neuronal somata in some brain regions (e.g., basal forebrain, caudate nucleus, thalamus). Under similar experimental conditions, we only occasionally detected faintly stained QPRT-i punctate structures in association with hippocampal neuronal somata in the present study.

At the ultrastructural level, the cytosol and mitochondrial membrane profiles were found to be strongly $3 \mathrm{HAO}-\mathrm{i}$, while only weak, or no, staining of lysosomes, Golgi apparatus, multivesicular bodies, and cisterns of the endoplasmic reticulum was observed. Ultrastructural analysis of QPRT-i cells, on the other hand, revealed a partly different intracellular distribution of QUINs catabolic enzyme. Thus, in some of the QPRT-i cells, the diffuse cytoplasmic staining was supplemented by intensely immunoreactive lysosome-like bodies scattered throughout the cytosol. These findings demonstrate that 3HAO-i and QPRT-i are in part associated with different organelles within the cells.

A striking observation in the present study was that $3 \mathrm{HAO}-\mathrm{i}$ cells greatly outnumbered those containing QPRT-i. While it cannot be ruled out that QPRT is present in all glial cells, but

\section{$\leftarrow$}

Figure 6. $A-D$, Color photomicrographs showing colocalization of GFAP (black-blue color; small arrows) and 3HAO (brown reaction product in $A$ ) or QPRT (brown reaction product in $B-D$ ) immunoreactivity. Photomicrographs in $A, B$, and $D$ are from different parts of Ammon's horn, while $C$ is from area dentata. Large arrows in B, GFAP-positive cells lacking QPRT-i. D, Examples of cells containing both GFAP and QPRT (large arrow) or GFAP or QPRT (crossed arrow) alone. C, A GFAP-positive (small arrows) cell containing QPRT-i in the molecular layer of area dentata-i shown in $C$. Bar, $10 \mu \mathrm{m}$. 
in somc instances cscapcs immunocytochemical detection because of excessively low levels of enzyme protein, the presence of a relatively small number of QPRT-i cells is certainly in line with biochemical findings demonstrating low QPRT activity in the rat hippocampus (Foster et al., 1985). Moreover, the present immunohistochemical analysis of $3 \mathrm{HAO}-\mathrm{i}$ cells is in agreement with the detection of high $3 \mathrm{HAO}$ activity in rat hippocampal homogenates (Foster et al., 1986). Finally, the present observations are in accordance with the results from recent studies performed in lesioned hippocampi, in which substantial increases in both $3 \mathrm{HAO}$ and QPRT activities were found following ibotenate-induced neuronal degeneration (Speciale and Schwarcz, 1987). Biochemical assessment had, therefore, previously led us to assume that $3 \mathrm{HAO}$ activity, in order to prevent the production of (toxic quantities of) QUIN, must be under the stringent control in vivo either of its substrate's or other regulatory mechanisms' bioavailability (Schwarcz et al., 1987). The present finding that not all QPRT-containing cells harbor 3 HAO (and vice versa) may have further implications for hippocampal QUIN function. It thus seems possible that the biosynthesis of QUIN at times takes place in cells different from those responsible for its catabolism. By inference, QUIN can be expected to be expelled into the extracellular space (where it can interact with NMDA receptors; Stone and Perkins, 1981) and subsequently enter (actively or passively) QPRT-containing cells.

Future studies will also have to explore the precise nature of the mechanisms governing the extracellular concentration of QUIN in view of its toxic potential. QUIN is present in normal cerebrospinal fluid in low concentration (Schwarcz et al., 1987), and increases may occur solely under pathological conditions. In this regard, it is noteworthy that QPRT-i cells exclusively are often seen in close association with neuronal somata, where they could serve a detoxifying role. Since an overabundance of QUIN has been hypothetically linked to the pathogenesis of a spectrum of neuropsychiatric disorders involving the hippocampal formation (see the introduction), it will be of importance to assess whether similar anatomical arrangements of 3HAO- and QPRT-containing cells also exist in the human hippocampus. Such studies are currently in progress.

\section{References}

Berod, A., B. K. Hartman, and J. F. Pujol (1981) Importance of fixation in immunohistochemistry: Use of formaldehyde at variable $\mathrm{pH}$ for the localization of tyrosine hydroxylase. J. Histochem. Cytochem. 29: 844-850.

Croucher, M. J., J. F. Collins, and B. S. Meldrum (1982) Anticonvulsant action of excitatory amino acid antagonists. Science 216:899901.

Delgado-Escueta, A. V., A. A. Ward, Jr., D. M. Woodbury, and R. J. Porter (1986) Basic Mechanisms of the Epilepsies: Molecular and Cellular Approaches. Advances in Neurology, vol. 44, Raven, New York.

Falconer, M. A., D. Hill, A. Meyer, W. Mitchell, and D. A. Pond (1955) Treatment of temporal lobe epilepsy by temporal lobectomy: A survey of findings and results. Lancet 1: 827-835.

Foster, A. C., and C. E. Fagg (1984) Acidic amino acid binding sites in mammalian neuronal membranes: Their characteristics and relationship to synaptic receptors. Brain Res. Rev. 7: 103-164.

Foster, A. C., B. S. Zinkand, and R. Schwarcz (1985) Quinolinic acid phosphoribosyltransferase in rat brain. J. Neurochem. 44: 446-454.

Foster, A. C., R. J. White, and R. Schwarcz (1986) Synthesis of quinolinic acid by 3 -hydroxyanthranilic acid oxygenase in rat brain tissue in vitro. J. Neurochem. 47: 23-30.

Gholson, R. K., I. Ueda, N. Ogasawara, and L. M. Henderson (1964)
The enzymic conversion of quinolinate to nicotinic acid mononucleotide in mammalian liver. J. Biol, Chem. 239: 1208-1214.

Hsu, S. M., L. Raine, and H. Fanger (1981) Use of avidin-biotinperoxidase complex (ABC) in immunoperoxidase techniques: A comparison between $\mathrm{ABC}$ and unlabelled antibody (PAP) procedures. J. Histochem. Cytochem. 29: 577-580.

Johansson, O., and J. Backman (1983) Enhancement of immunoperoxidase staining using osmium tetroxide. J. Neurosci. Methods 7: 185-193.

Köhler, C., and R. Schwarcz (1981) Monosodium glutamate: Increased neurotoxicity after removal of neuronal reuptake sites. Brain Res. 221: 485-491.

Köhler, C., E. Okuno, P. R. Flood, and R. Schwarcz (1987) Quinolinic acid phosphoribosyltransferase: Preferential localization in the rat brain visualized by immunocytochemistry. Proc. Natl. Acad. Sci. USA 54: 3491-3495.

Lapin, I. P. (1981) Kynurenines and seizures. Epilepsia 22: 257-265.

Lapin, I. P., I. B. Prakhie, and I. P. Kiseleva (1982) Excitatory effects of kynurenine and its metabolites, amino acids and convulsants administered into brain ventricles: Differences between rats and mice. J. Neural Transm. 54: 229-238.

MacLean, I., and P. Nakane (1974) Periodate-lysine paraformaldehyde fixative: A new fixative for immunoelectronmicroscopy. J. Histochem. Cytochem. 22: 1077-1083.

Moroni, F., G. Lombardi, V. Carola, and G. Moneti (1984) The excitotoxin quinolinic acid is present and unevenly distributed in the rat brain. Brain Res. 295: 352-355.

Nishizuka, Y., and O. Hayaishi (1963) Studies on the biosynthesis of nicotinamide adenine dinucleotide. I. Enzymatic synthesis of niacin ribonucleotides from 3-hydroxy-anthranilate oxygenase from beef kidney. J. Biol. Chem. 238: 3369-3377.

Okuno, E., and R. Schwarcz (1985) Purification of quinolinic acid phosphoribosyltransferase from rat liver and brain. Biochim. Biophys. Acta $841: 112-119$.

Okuno, E., C. Köhler, and R. Schwarcz (1987) Rat 3-hydroxyanthranilic acid oxygenase: Purification from the liver and immunocytochemical localization in the brain. J. Neurochem. 49: 771-780.

Perkins, M. N., and T. W. Stone (1983) Pharmacology and regional variations of quinolinic acid-evoked excitation in the rat central nervous system. J. Pharmacol. Exp. Ther. 226: 551-557.

Peters, A., S. L. Palay, and H. Webster (1976) The Fine Structure of the Nervous System. The Neurons and Supporting Cells, Saunders, Philadelphia, London.

Schwarcz, R., and Y. Ben-Ari (1986) Excitatory Amino Acids and Epilepsy. Plenum, New York.

Schwarcz, R., and C. Köhler (1983) Different vulnerability of central neurons of the rat to quinolinic acid. Neurosci. Lett. 38: 85-90.

Schwarcz, R., and B. Meldrum (1985) Excitatory amino acid antagonists provide a therapeutic approach to neurological disorders. Lancet 2: 140-143.

Schwarcz, R., J. F. Collins, and D. A. Parks (1982) $\alpha$-Amino- $\omega$-phosphonocarboxylates block ibotenate but not kainate neurotoxicity in rat hippocampus. Neurosci. Lett. 33: 85-90.

Schwarcz, R., G. S. Brush, A. C. Foster, and E. D. French (1984) Seizure activity and lesions following intrahippocampal injection of quinolinic acid. Exp. Neurol. 84: 1-17.

Schwarcz, R., C. Speciale, E. Okuno, E. D. French, and C. Köhler (1986) Quinolinic acid: A pathogen in seizure disorders. In Excitatory Amino Acids and Epilepsy, R. Schwarcz and Y. Ben-Ari, eds., pp. 697-707, Plenum, New York.

Schwarcz, R., E. Okuno, C. Speciale, C. Köhler, and W. D. Whetsell, Jr. (1987) Neuronal degeneration in animals and man: The quinolinic acid connection. In Neurotoxins and their Pharmacological Implications, P. G. Jenner, N. G. Bowery, J. E. Cremer, J. O. Dolly, and M. Sandler, eds., pp. 19-33, Raven, New York.

Sommer, W. (1880) Erkrankungen des Ammonshorns als aetiologisches Moment der Epilepsie. Arch. Psychiat. Nervenkr. 10: 631-675.

Speciale, C., and R. Schwarcz (1987) Effect of systemic kainate administration on cerebral quinolinic acid metabolism in the rat. Exp. Neurol. (in press).

Stone, T. W., and M. N. Perkins (1981) Quinolinic acid: A potent endogeneous excitant at amino acid receptors in CNS. Eur. J. Pharmacol. 72: 411-412.

Vaughan, D. W. (1984) The structure of neuroglial cells. In Cerebral Cortex, vol. 2: Functional Properties of Cortical Cells, E. G. Jones and 
A. Peters, eds., pp. 285-325, Plenum Press, New York

Wolfensberger, M., V. Amsler, M. Cuénod, A. C. Foster, W. U. Whetsell, Jr., and R. Schwarcz (1983) Identification of quinolinic acid in rat and human brain tissue. Neurosci. Lett. 41: 247-252.
Wong, E. H. F., J. A. Kemp, T. Priestley, A. R. Knight, G. N. Woodruff and $L$. L. Iversen (1986) The anticonvulsant MK-801 is a potent $N$-methyl-D-aspartate antagonist. Proc. Natl. Acad. Sci. USA 83:7104 7108 . 